\title{
Ambientes distribuídos em Realidade Virtual como suporte à Aprendizagem Cooperativa para a Resolução de Problemas
}

\author{
Luciano Ferreira Silva, DCC/UFRR, luciano@mat.ufu.br \\ Alexandre Cardoso, PPGEE/UFU, alexandre@ufu.br \\ Elise B. Mendes, FACED/UFU, elise@ufu.br \\ Edgard A. Lamounier Jr. PPGEE/UFU, lamounier@ufu.br \\ Eduardo Kojy Takahashi, INFIS/UFU, ektakahashi@ufu.br
}

\begin{abstract}
Resumo: Um grande número de pesquisadores acredita que a resolução de problemas é inerente a grande parte dos processos de ensino e aprendizagem. Considera-se ainda, que esta metodologia apresenta uma maior eficácia quando aplicada cooperativamente. Neste contexto, este artigo apresenta uma proposta multidisciplinar que viabiliza o uso da Realidade Virtual distribuída para oferecer suporte à resolução de problemas por meio da aprendizagem cooperativa. Para tal, são considerados os fundamentos da aprendizagem por experimentação e teorias e princípios pedagógicos construtivistas.

Palavras-Chave: Realidade Virtual, Resolução de Problemas, Aprendizagem Cooperativa.

\section{Distributed environment in Virtual Reality as support the Cooperative Learning for the Problems Solving}

Abstract: A great number of researchers believe that problem resolution is inherent from the great part of the teaching and learning processes. It is considered that this methodology presents a better effectiveness when applied cooperatively. In this context, this paper presents a multidisciplinary proposal that allows the use of Virtual Reality to offers support to the resolution of problems through the cooperative learning. For this, the learning foundations will be considered by experimentation and theories and constructivities pedagogic principles.

Key-words: Virtual reality, Problems Solving, Cooperative Learning.

\section{Introdução}

Nos últimos anos, ampliaram-se as pesquisas que têm como intuito promover a aprendizagem cooperativa e colaborativa em ambientes computacionais, bem como o desenvolvimento de competências cognitivas, tais como a resolução de problemas (Aaas, 2001). Inúmeros autores consideram que essas novas pesquisas devem contrapor-se aos estudos que utilizam as novas tecnologias apenas para transmitir e tornar disponível as informações aos estudantes. Dessa forma, as novas propostas de ensino buscam proporcionar cenários de aprendizagem que estimulem o desenvolvimento da autonomia intelectual e isto é possível por meio de processos de aprendizagem que promovem o raciocínio e a resolução de problemas de forma coletiva (Barros, 1994).

Algumas estratégias de ensino associadas à mídia moderna podem oferecer suporte a essa aprendizagem, uma vez que transformam as formas de transmissão e assimilação do conhecimento, influenciando a maneira de conceber e realizar a formação de professores e alunos (Silveira e Arruda, 1998). Dentre elas, a Realidade Virtual (RV) apresenta-se como uma tecnologia que oferece prósperas contribuições ao desenvolvimento de aplicações voltadas para o ensino. De acordo com Barros e Kelner (2003) as razões para utilizar RV no ensino são diversas, destacando-se, por exemplo, a

V. $7 \mathrm{~N}^{\mathrm{o}}$ 3, dezembro, 2009 
maior motivação do usuário; pois RV é uma forma com a qual as pessoas visualizam, manipulam e interagem com computadores e dados extremamente complexos. Além disso, a RV possibilita a criação de ambientes virtuais interativos e intuitivos, proporcionando a manipulação direta de seus objetos que respondem às ações do usuário, estimulando sua participação ativa.

Neste contexto pode-se observar que a resolução de problemas é uma importante estratégia de ensino, que está envolvida em uma grande parte das situações de aprendizagem. Percebe-se, também, que o sucesso desta metodologia está profundamente relacionado com a sua ligação a modelos pedagógicos que contemplam a aprendizagem cooperativa (Barros, 1994). Verifica-se ainda, que a RV oferece vantagens significativas para a sua aplicação em processos de ensino e aprendizagem, e, além disso, ela pode oferecer suporte a aprendizagem cooperativa por meio de ambientes virtuais distribuídos.

Considerando os fatores supracitados, que norteiam as motivações desta pesquisa, o objetivo deste trabalho é propor e validar estratégias computacionais e metodologias pedagógicas associadas ao desenvolvimento de ambientes de RV, em sistemas distribuídos, para suporte à resolução de problemas por meio da aprendizagem cooperativa. Para tanto, deve-se considerar as teorias e os princípios pedagógicos construtivistas e os fundamentos de experimentação por meio de laboratórios virtuais. Como estudo de caso, optou-se por desenvolver o projeto no âmbito da Física.

A próxima seção aborda os fundamentos teóricos envolvidos nesta pesquisa, os quais são indispensáveis para a perfeita compreensão deste trabalho.

\section{Fundamentos teóricos}

Esta seção apresenta os subsídios pedagógicos e cognitivos que formam o aporte teórico do presente trabalho. Para tanto, são aduzidos conceitos e teorias envolvendo RV, cognição, objetos de aprendizagem, ambientes cooperativos, resolução de problemas, entre outros.

\subsection{Realidade Virtual e Educação}

Existem diversas definições sobre RV, as quais envolvem aspectos científicos variados. Uma abordagem que apresenta uma visão geral e que sintetiza as várias discussões de RV é apresentada por Kirner e Siscoutto (2007): "Realidade Virtual é uma interface avançada para aplicações computacionais, que permite ao usuário navegar, manipular e interagir, em tempo real, com um ambiente tridimensional gerado por computador". É importante complementar, que a interação do usuário com o ambiente virtual é um dos aspectos mais importantes da interface baseada em RV, visto que ela está relacionada com a capacidade do computador detectar e reagir às ações do usuário, promovendo alterações na aplicação (Bowman et al., 2005).

Em RV o usuário deve ter a impressão de estar atuando no ambiente virtual, apontando, pegando, manipulando e executando outras ações sobre os objetos virtuais, em tempo-real. Esta experiência virtual permite que o usuário possa aprender a partir da experimentação e descoberta. Tal fato é de extrema importância, pois como ressalta Ficheman (2006), a educação pode ser vista como um processo de descoberta, exploração e de observação, além de eterna construção do conhecimento.

\subsection{Aprendizagem cooperativa}

Existem diversas teorias que abordam a aprendizagem coletiva e destacam a importância do estudo e do trabalho em grupo no processo de ensino e aprendizagem, e 
no desenvolvimento sociocultural dos aprendizes. Em levantamentos realizados em relação às recordações de experiências de aprendizagem, constatou-se que a maioria das pessoas lembra de situações envolvendo a aprendizagem que requer alguma ação coletiva (Larocque e Faucon, 1997). Estes estudos sobre a cooperação e colaboração entre aprendizes partem da idéia de que o conhecimento é resultante de um consenso entre membros de uma comunidade de conhecimento, algo que as pessoas constroem pela comunicação, trabalhando juntas direta ou indiretamente e chegando a coordenações de pontos de vista (Torres, Alcantara e Irala, 2004).

Frequentemente encontram-se obras nas quais os termos cooperação e colaboração são usados como sinônimos, porém, cada um destes termos, ao longo dos anos, desenvolveu distinções próprias e diferentes aplicações práticas. Alguns pesquisadores acreditam que o termo cooperação é mais abrangente, com distinções hierárquicas de ajuda mútua, ao passo que na colaboração existe um objetivo comum entre as pessoas que trabalham em conjunto sem uma hierarquia (Nitzke et al., 1999). Muitos autores na literatura atual definem a aprendizagem cooperativa como uma aprendizagem mais estruturada, com técnicas de sala de aula mais prescritivas e com regras mais definidas de como deve se processar a interação entre os alunos, se comparada com a aprendizagem colaborativa (Oxford, 1997). Considerando tais definições o presente trabalho adota a expressão "aprendizagem cooperativa".

Em termos tecnológicos ambientes distribuídos em RV oferecem suporte ao trabalho cooperativo, possibilitando o acesso simultâneo de multi-usuários a um ambiente virtual.

\subsection{Resolução de problemas}

A humanidade confronta-se com a palavra "problema" há milhares de anos. No entanto, a sua definição propicia a inferência de diversas interpretações. Por exemplo, problema pode ser considerado como um estado subjetivo da mente inerente a cada indivíduo, um desafio, uma situação não resolvida, cuja resposta não é imediata, que resulta em reflexão e uso de estratégias conceituais e procedimentais, provocando uma mudança nas estruturas mentais (Costa e Moreira, 1995).

Os processos cognitivos realizados pela mente humana no tratamento e na resolução de problemas despertaram interesses no campo da Psicologia, que iniciou intensos estudos sobre tais processos. Estes estudos demonstraram que o indivíduo adquire um aprendizado decorrente destes processos cognitivos. Bono (1973) acredita que processos criativos e de aprendizagem devem iniciar-se efetivamente por meio de um problema. Com isto, esta área de conhecimento tornou-se atrativa e aplicável ao ensino e serviu como suporte para a criação de metodologias de ensino denominadas resolução de problemas. Essas metodologias têm despertado o interesse de pesquisadores de varias áreas do ensino como, por exemplo, Biologia, Matemática, Física, etc. Este interesse pode ser atribuído ao fato de que a resolução de problemas contribui significativamente para as atividades de raciocínio e pensamento exigidos nestas disciplinas (Costa e Moreira, 1995).

Alguns pesquisadores consideram a resolução de problemas como "um processo pelo qual o aprendiz descobre uma combinação de regras anteriormente aprendidas, que ele pode aplicar para atingir uma solução para uma situação problemática nova" (Gagné, 1965). Este processo deve favorecer a aprendizagem significativa na medida em que propicia uma reorganização da informação e do conhecimento armazenado na estrutura cognitiva do sujeito (Novak e Tyler, 1986).

\subsection{Desenho pedagógico do projeto}


A integração da teoria de aprendizagem cooperativa e da resolução de problemas requer o desenvolvimento de um ambiente computacional de aprendizagem que estimule os conhecimentos conceitual, procedimental e metacognitivo. Estes conhecimentos podem ser estimulados por meio da RV em sistemas distribuídos, já que existe a possibilidade da experimentação com objetos virtuais, da interação e da comunicação para solucionar problemas abertos pela coordenação de pontos de vistas (cooperação). Assim, conclui-se que o desenho pedagógico do projeto deve estar no âmbito de três áreas de conhecimento: a RV (com ambientes computacionais em RV), a aprendizagem cooperativa (suportada por rede de computares) e a resolução de problemas (aplicados à Física).

A metodologia de ensino e o sistema de avaliação do desenho pedagógico, proposto para o projeto, são fundamentados nos estudos de resolução de problemas desenvolvidos pela área de Educação (Gargiulo e Mendes, 2004). Nesse estudo, os autores fazem uma integração das teorias de Polya, Pozo, Bransford e Stein, Backer e Mayer, e Kapa em um modelo geral, que tem como matriz de compreensão a taxonomia revisada dos objetivos cognitivos $\mathrm{O}$ modelo de resolução de problemas obtido possui três operações não-lineares, que são: contato inicial (constituído de três processos fundamentais: a identificação do problema, a representação mental do problema e a geração de hipóteses); solução (formada de cinco processos fundamentais: a busca e exploração de estratégias, o projeto de solução, a previsão de resultados, a aplicação e o gerenciamento da aplicação); e avaliação (constituída de dois processos fundamentais: a crítica da solução e a entrega da solução). Nesta metodologia, os processos cognitivos e conhecimentos necessários a cada estágio da resolução dos problemas foram explicitamente definidos, diferentemente dos modelos de solução revisados, que focam mais nos passos de solução do que nos detalhes das tarefas.

\section{Trabalhos correlatos}

Com intuito de avaliar o estado das pesquisas concernentes ao presente trabalho, foram analisadas ferramentas computacionais voltadas para o ensino, que se baseiam na resolução de problemas, e/ou aprendizagem cooperativa e/ou RV, sendo verificadas suas vantagens e limitações no processo de ensino e aprendizagem. Os trabalhos analisados foram:

- O projeto Andes, desenvolvido pela University of Pittsburgh em parceria com a United States Naval Academy, com apoio do Office of Naval Research por meio do Programa de Ciência Cognitiva (Cognitive Science Program). Este projeto estuda o uso de metodologias de resolução de problemas aplicadas ao ensino de Física. Para tanto, uma de suas principais metas é modificar o modo com que os alunos efetuam suas tarefas de casa; pretende-se substituir o método tradicional de resolver exercícios usando "lápis e papel" pelo método no qual o aluno utiliza o computador para tal fim. com o objetivo de verificar se esta mudança aumenta o aprendizado do estudante (Vanlehn et al., 2005).

- O eJournal (The Electronic Laboratory Journal), norteado pela Manchester Metropolitan University, que visa o desenvolvimento de um ambiente colaborativo e cooperativo voltado para o ensino por meio da experimentação baseada em Web. De acordo com o projeto eJornal, foram desenvolvidas varias ferramentas para apoiar a colaboração entre estudantes. Estas ferramentas incluem principalmente artifícios para compartilhar documentos e habilitar discussões (Fakas, Nguyen e Gillet, 2005).

- O WebTOP, criado pela University of State Mississipi, possui o intuito de ensinar Óptica e foi totalmente desenvolvido em VRML e Java. O sistema é 
composto por dezesseis módulos diferentes, envolvendo ondas, ótica geométrica, reflexão e refração, polarização, interferência, difração, distribuídos em oito áreas da Óptica. Cada módulo possui simulações computacionais interativas, animadas e em 3D para apresentação de fenômenos físicos, sendo utilizados applets Java para controlar interações do usuário com os ambientes de experimento (Mzoughi et al., 2005).

- O projeto Supporting Distance Learners For Collaborative Problem Solving, desenvolvido pela Universidad Nacional de Educación a Distancia - UNED em parceria com a Universitat Politècnica de Catalunya - UPC. Esta pesquisa focaliza, essencialmente, o estudo de métodos de resolução de problemas por meio de sistemas colaborativos, em particular, aplicados ao desenvolvimento de software. Para tanto, a arquitetura e construção do sistema foram baseadas em observações e discussões de estudantes da UNED e UPC, assim como, em suas anotações usadas para descrever o domínio de objetos de aprendizagem, e descrições de problemas e instâncias necessárias para a sua solução (Verdejo, Barros e Abad, 1998).

- O projeto ARCA, acrônimo de Ambiente de Realidade Virtual Cooperativo de Aprendizagem, está sendo desenvolvido por três áreas de ensino da Universidade Federal do Rio Grande do Sul - UFRGS: Psicologia, Educação e Informática, coordenado pelo Programa de Pós-Graduação em Informática na Educação (Ufrgs, 2008). Este projeto propõe-se a desenvolver um ambiente de ensino e aprendizagem que, apoiado pela Internet, possa atuar como instrumento no auxílio a uma prática pedagógica diferenciada. $\mathrm{O}$ seu ambiente visa criar condições para uma aprendizagem significativa por meio de um ambiente desenvolvido em RV que permite a cooperação.

- O DEVRL, acrônimo de Distributed Extensible Virtual Reality Laboratory, desenvolvido pela University of Lancaster, visa possibilitar à alunos de diferentes localidades do Reino Unido trabalhar em um mesmo espaço virtual e realizar tarefas comuns. Duas aplicações teste foram desenvolvidas: o Nottingham, que permite ao usuário navegar localmente por representações 3D das informações contidas no espaço virtual e o Lancaster, que possibilita a criação de uma sala de jogos virtual. Esta sala tem como objetivo fornecer aos usuários um sistema distribuído que possibilita aplicações em RV baseadas em colaboração, e que permitam a experimentação das propriedades físicas por meio de simulações de jogos (Mel et al., 1996).

- O projeto NICE, acrônimo de Narrative Immersive Constructionist/Collaborative Environments, desenvolvido pela University of Illinois at Chicago e tem como intuito aplicar RV para a criação de ambientes educacionais para crianças. Ele foi projetado com base em teorias construtivistas, proporcionando aos seus usuários uma motivação, por meio de uma narrativa, para construírem mundos virtuais colaborativamente (Roussos et al., 1997).

- O Construct3D, desenvolvido pelo Institute of Software Technology and Interactive Systems na Vienna University of Technology e pelo Institute for Computer Graphics and Vision na Graz University of Technology. Este projeto visa à criação de uma ferramenta que possibilite a construção geométrica tridimensional, sendo que, ela está direcionada especificamente para o ensino de matemática e geometria (Kaufmann e Schmalstieg, 2006). 


\section{Arquitetura do sistema}

A arquitetura do sistema proposta pela equipe multidisciplinar visa oferecer suporte para professores e alunos no processo de ensino e aprendizagem, o qual se baseia em teorias da aprendizagem cooperativa e resolução de problemas, aplicadas a sistemas distribuídos de RV. Para tanto, deve-se possibilitar ao professor gerenciar e acompanhar as atividades do aluno, permitindo que ele proponha atividades experimentais (problemas) e tenha retornos quanto à efetividade das mesmas. Este processo permite ao professor verificar como o aluno está trabalhando com as atividades sugeridas e como está ocorrendo sua aprendizagem mediante elas. Deste modo, o professor terá uma noção avaliativa do processo de aprendizagem do aluno, o que facilitará a sugestão de novas atividades e o direcionamento do estudo do aluno. $\mathrm{O}$ aluno, por sua vez, deve ter acesso a laboratórios virtuais, desenvolvidos por meio de $\mathrm{RV}$, nos quais poderá resolver problemas com apoio de experimentos virtuais. O aluno deve, ainda, enviar a solução de sua atividade e suas dúvidas no decorrer do processo de resolução, para análise dos professores. A Figura 1 ilustra o diagrama da arquitetura do sistema.

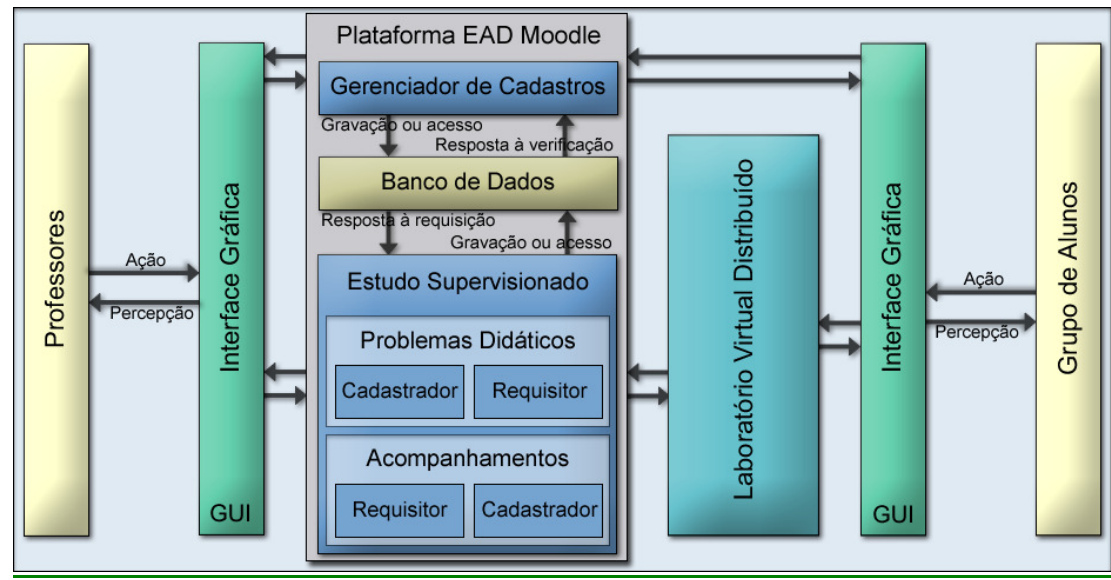

Figura 1: Arquitetura do sistema.

O fator preponderante que influenciou na escolha da plataforma Moodle para complementar este trabalho, foi o fato que o público alvo para a avaliação do sistema já utilizavam esta plataforma há dois anos aproximadamente para realizar suas atividades acadêmicas. Neste contexto, os usuários a serem avaliados apresentariam menores dificuldades em trabalhar com esta plataforma, uma vez comparada com um sistema totalmente novo para eles. Esta ambientação é de grande importância para a avaliação do sistema, pois pode influenciar diretamente os resultados finais obtidos por meio deste trabalho. A próxima seção apresenta detalhes do desenvolvimento do Laboratório Virtual Distribuído desta arquitetura.

\section{Detalhes de desenvolvimento do protótipo virtual}

O Laboratório Virtual Distribuído (LVD) consiste em um ambiente distribuído em RV com um cenário de um laboratório de Física. O ambiente virtual do LVD é composto por uma série de objetos de aprendizagem, como por exemplo, uma balança de precisão, um calorímetro, um becker, um bico de bunsen, um pistão didático, um trilho de ar, cronômetros digitais, termômetro, trena e diversos tipos de pesos. O modelo comportamental destes objetos foi desenvolvido realisticamente, obedecendo até mesmo os erros do mundo real, como por exemplo, o erro mecânico de medição da balança, o erro de medição do termômetro devido a perda de calor para o ar, etc. 
Para o desenvolvimento dos objetos virtuais foi utilizada a linguagem VRML, acrônimo de Virtual Reality Modeling Language, que possibilita a criação de mundos virtuais tridimensionais, com alta qualidade, aplicáveis à Internet. Para a visualização utilizou o Xj3D, uma ferramenta Java, com o código aberto, criada para a visualização e a manipulação de conteúdos escritos em VRML e X3D. A Figura 2 apresenta a interface de dois ambientes do LVD conectados via localhost.

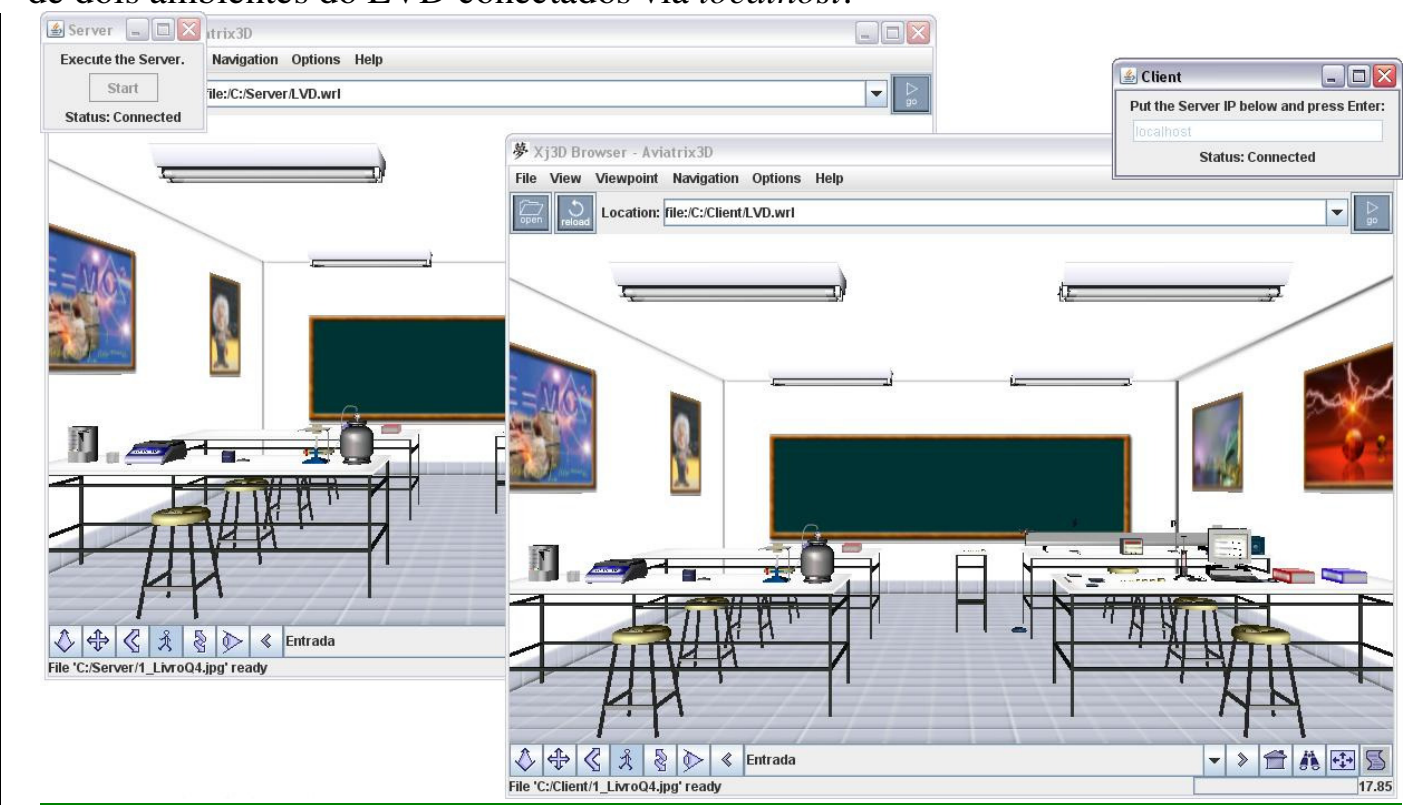

Figura 2: Ambientes LVD conectados via localhost.

Para realizar a distribuição do LVD foi necessária a criação de uma arquitetura própria para tal fim e algoritmos específicos, levando em consideração que a linguagem VRML é estática e baseada em eventos. A arquitetura desenvolvida encontra-se ilustrada na Figura 3.

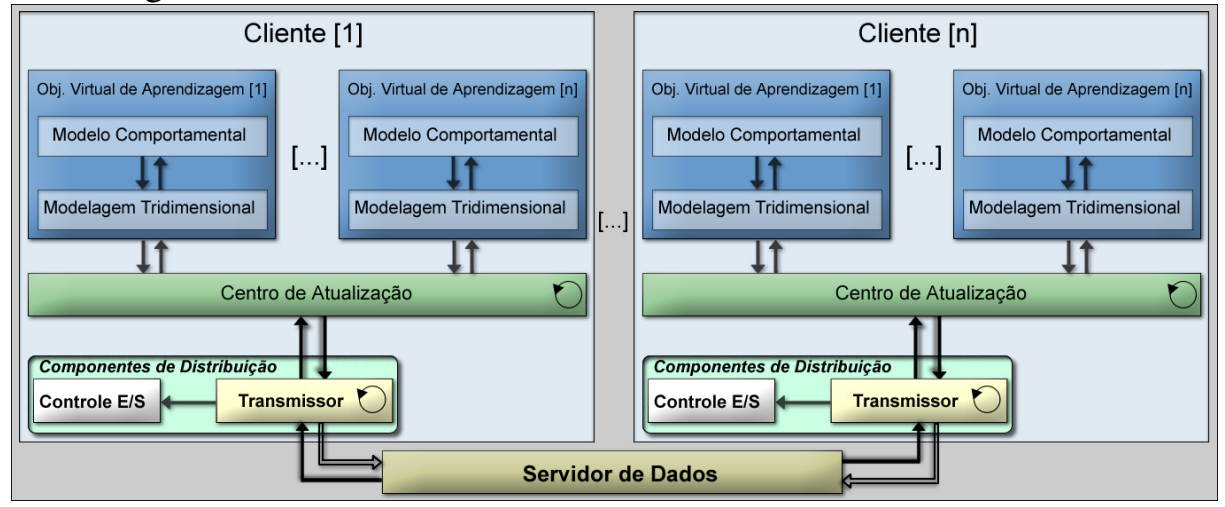

Figura 3: Arquitetura de distribuição do LVD.

Para efetivar a comunicação entre os ambientes do LVD utilizou-se a tecnologia sockets em Java, pois a mesma apresenta resultados satisfatórios quanto ao seu desempenho e linguagem VRML permite o uso de ferramentas Java por meio do nó Script.

\section{Avaliação do sistema}

A metodologia de avaliação utilizada neste projeto consiste em uma seqüência de passos que visa o alcance de resultados condizentes, veridicamente, com a situação real avaliada. Para tanto, faz-se necessária à adoção de técnicas e procedimentos 
avaliativos adequados para minimizar os fatores que influenciam, negativamente ou positivamente, o processo de validação do sistema.

O primeiro passo da metodologia de avaliação é separar uma amostra $\lambda$ de uma população $\Omega$ de alunos aptos aos testes, por um processo de amostragem probabilístico aleatório simples sem reposição. A amostra $\lambda$ do projeto foi composta por 84 alunos do ensino médio.

O segundo passo da metodologia consiste em apresentar aulas expositivas do assunto a ser avaliado para a amostra $\lambda$, no caso do presente projeto foram ministras aulas sobre mecânica, em específico o estudo de movimentos retilíneos uniformes ou variados e leis de Newton. No terceiro passo ocorre a aplicação de um teste básico sobre os conceitos aprendidos nas aulas expositivas do segundo passo.

No quarto passo a amostra $\lambda$ é dividida em três grupos, $\alpha, \beta$ e $\gamma$, com quantidades iguais de elementos (28 indivíduos cada). Este procedimento precisa ser realizado de modo que $\alpha, \beta$ e $\gamma$ preservem as características de $\lambda$. Para tanto, utiliza-se como parâmetro a nota de cada indivíduo no teste do terceiro passo. Após, considerando $\mu \alpha$, $\mu \beta$, e $\mu \gamma$ como sendo, respectivamente, a média de $\alpha, \beta$ e $\gamma$, e conhecendo-se os seus respectivos desvios padrões $\sigma \alpha, \sigma \beta$ e $\sigma \gamma$, devem-se então ocorrer que $\mu \alpha, \mu \beta$ e $\mu \gamma$ são estatisticamente iguais a $\mu \lambda$. Esta igualdade estatística pode ser verificada por meio de um teste de hipóteses de diferença de médias.

No quinto passo os grupos $\alpha, \beta$ e $\gamma$ são separados fisicamente e cada um deles passa a trabalhar de forma específica. As atividades para os três grupos são aplicadas, de modo que, os indivíduos do grupo $\alpha$ resolverão estas atividades de forma individual e sem utilizarem o ambiente virtual do LVD; os indivíduos do grupo $\beta$ resolverão suas atividades também de maneira individual, porém, com o auxílio do ambiente LVD; os indivíduos do grupo $\gamma$, por sua vez, realizarão as suas atividades usando o LVD de maneira cooperativa, em grupos de duas pessoas escolhidas aleatoriamente por meio de sorteio. As atividades aplicadas são as mesmas para os três grupos e são compostas efetivamente por problemas. Estes problemas propõem inicialmente uma situação a qual deriva novos tópicos problemas, sendo que, cada um destes tópicos é desenvolvido em correspondência com uma das fases do modelo de resolução de problemas proposto neste projeto.

As estratégias supracitadas visam permitir a avaliação de cada etapa da resolução de problemas em relação às tecnologias e metodologias pedagógicas propostas neste trabalho. Para tanto, entende-se que deve ser realizada uma avaliação de maneira comparativa, na qual uma primeira amostra é testada sem a influência da RV, uma segunda utiliza a RV de forma individual e uma terceira utilizada a RV cooperativamente. Como os grupos avaliados possuem as mesmas características, a comparação dos resultados torna-se mais confiável, sendo possível verificar em quais etapas da resolução de problemas a RV contribuiu e ainda, em quais de seus aspectos o uso cooperativo da RV influencia.

Para possibilitar a avaliação da evolução do aluno em resolver problemas no decorrer do processo avaliativo foi realizada uma classificação dos problemas desenvolvidos em termos de níveis de dificuldade de solução. Quanto maior o número de conceitos necessários para a resolução e elevada a complexidade do plano de solução maior será considerado o seu nível de dificuldade do problema. A análise dos resultados da avaliação de acordo com os níveis de dificuldade dos problemas permite verificar comparativamente o quanto cada amostra aproveitou, de maneira benéfica, o processo de resolução para aprimorar os seus conhecimentos e a sua capacidade de generalização e de aplicação de estratégias e teorias. 
Por fim, no sexto passo são catalogados os resultados das avaliações referentes a cada etapa do modelo de resolução de problemas, colocando-os em gráficos para uma melhor visualização, análises e conclusões.

\section{Conclusão}

Ao analisar projetos nas áreas de pesquisa da resolução de problemas, aprendizagem cooperativa e RV pode-se perceber certa carência de trabalhos com focos nas interseções destas áreas. Neste contexto, a presente pesquisa se dispôs a gerar estudos de cunho teórico e tecnológico para criar alternativas de qualidade que contribuam para o processo ensino e aprendizagem em grande extensão nas escolas. Dessa forma, a integração dos estudos teóricos sobre desenhos pedagógicos relacionados à cooperação e solução de problemas aliados à realidade virtual, possivelmente possam contribuir com os objetivos educacionais mais amplos de estimular o pensamento formal e a capacidade de resolução de problemas de forma cooperativa.

Os ambientes distribuídos desenvolvidos (LVD) apresentaram um alto desempenho e mostraram-se adequados para a efetivação dos testes em relação à aprendizagem cooperativa. Porém, para obter informações mais completas, concretas e contundentes sobre a qualidade da arquitetura e dos algoritmos de distribuição propostos neste projeto, faz-se necessária à realização de uma avaliação específica quanto aos parâmetros qualitativos de uma distribuição, como por exemplo, desempenho, escalabilidade, tolerância a falhas, segurança, transparência, etc.

Como resultado da avaliação do sistema observou-se que a amostra que trabalhou com resolução de problemas cooperativamente no ambiente de RV conseguiu um melhor desempenho que as outras duas amostras, que trabalharam de forma individual sem o auxílio tecnológico e com apoio do ambiente LVD em RV para a resolução de problemas. As amostras que utilizaram os ambientes em RV conseguiram melhores desempenhos durante o aumento da complexidade dos problemas, o que pode indicar que a RV contribuiu para estas amostras ativarem os seus conhecimentos para desenvolverem estratégias mais elaboradas e resolverem problemas de maior complexidade.

\section{Agradecimentos}

Agradecemos a CAPES pelo suporte financeiro através da bolsa de doutorado, a Victor Gargiulo pela elaboração da metodologia de análise dos processos cognitivos de solução de Problemas e a todos que contribuíram de forma direta ou indireta para a realização deste trabalho.

\section{Referências}

AAAS. project 2061. Atlas of Science Literacy. Washington: American Association for the Advancement of Science 2001.

BARROS, L. A. Suporte a Ambientes Distribuídos para Aprendizagem Cooperativa . Universidade do Rio de Janeiro, 1994. Tese de Doutorado

BARROS, P. G. e J. KELNER. Simulação de tráfego, uma experiência com realidade virtual. In: VI Symposium on Virtual Reality. Ribeirão Preto - SP: 2003. 140-151 p. BONO, E. D. Lateral Thinking: Creativity Step by Step. H. R. Publishers. New York 1973. 
BOWMAN, D. A., et al. 3D User Interfaces: Theory and Practice. Boston: MA: Addison-Wesley. 2005

COSTA, S. S. C. D. e M. A. Moreira. Resolução de problemas I: diferença entre novatos e especialistas. Comissão Organizadora da IX Reunião de Educação em Física da Associação Argentina de Professores de Física. Salta - Argentina 1995.

FAKAS, G. J., A. V. NGUYEN e D. GILLET. The Electronic Laboratory Journal: A Collaborative and Cooperative Learning Environment for Web-Based Experimentation. Computer Supported Cooperative Work - Springer. 14: 189-216 p. 2005.

FICHEMAN, I. K., et al. Gruta Digital: um Ambiente de Realidade Virtual Imersivo Itinerante para Aplicações Educacionais. In: XVII Simpósio Brasileiro de Informática na Educação, Brasília: SBC, 2006. p.

GAGNÉ, R. M. The Conditions of Learning. New York: Holt, Rinehart and Winston. 1965

GARGIULO, V e MENDES, E. (2007) Análise e Integração de Teorias de Solução de Problemas segundo Objetivos Cognitivos. Relatório de Iniciação Científica. Bolsa FAPEMIG/ UFU (material impresso).

KAUFMANN, H. e D. SCHMALSTIEG. Designing Immersive Virtual Reality for Geometry Education. IEEE Virtual Reality. Alexandria, Virginia, USA: IEEE, 2006. $52-58 \mathrm{p}$.

KIRNER, C. e R. A. SISCOUTTO. Fundamentos de Realidade Virtual e Aumentada. In: C. Kirner e R. A. Siscoutto (Ed.). Realidade Virtual e Aumentada: Conceitos, Projeto e Aplicações: SBC, 2007.

LAROCQUE, D. e N. FAUCON. Me, myself and ... you? Collaborative learning: why bother? Teaching in the Community Colleges Online Conference - Trends and Issues in Online Instruction. Toronto, Ontario 1997.

MEL, S., et al. Distributed extensible virtual reality laboratory (DEVRL): a project for co-operation in multi-participant environments. Proceedings of the Eurographics workshop on Virtual environments and scientific visualization 96. Monte Carlo, Monaco: Springer-Verlag 1996.

MZOUGHI, T., et al. WebTOP: Web-based interactive 3D optics and waves simulations. International Journal of Continuing Engineering Education and LifeLong Learning, v.15, n.2, p.79-94. 2005.

NITZKE, J. A., et al. Avaliando aplicações para criação de ambientes de aprendizagem colaborativa. In: Simpósio Brasileiro de Informática na Educação. Curitiba, 1999. 303-310 p.

NOVAK, J. D. e R. W. Tyler. A Theory of Education. Cornell University Press. 1986

Oxford, R. L. Cooperative Learning, Collaborative Learning, and Interaction: Three Communicative Strands in the Language Classroom. The Modern Language Journal, v.81, n.4, p.443-456. 1997.

ROUSSOS, M., et al. NICE: combining constructionism, narrative and collaboration in a virtual learning environment. ACM SIGGRAPH Computer Graphics, v.31, n.3, p.62 - 63. 1997.

SILVEIRA, M. e L. ARRUDA. Desenvolvimento de um Sistema Multimídia para a Autoria e Apresentação de Cursos de Controle. 3rd Symposium Investigation and Development of Educational Software. Évora - Portugal, 1998. p.

TORRES, P. L., P. R. ALCANTARA e E. A. F. Irala. Grupos de Consenso: uma proposta de aprendizagem colaborativa para o processo de ensino-aprendizagem. Revista Diálogo Educacional, v.4, n.13, p.129-145. 2004.

UFRGS.ARCA. Disponível em: <http://penta.ufrgs.br/pgie/arca/arca.htm>. Acesso em: 19 out. 2009

V. $7 \mathrm{~N}^{\mathrm{o}}$ 3, dezembro, 2009 
VANLEHN, K., et al. The Andes Physics Tutoring System: Lessons Learned. International Journal of Artificial Intelligence in Education, v.15, n.3, p.1-47. 2005. VERDEJO, M. F., B. BARROS e M. T. Abad. Supporting Distance Learners for Collaborative Problem Solving. ED-MEDIA \& E-TELECOM, v.1, p.1407-1412. 1998. 